\title{
ON REGULAR-INVARIANCE OF CONTINUITY
}

\author{
R. G. ORI ${ }^{1}$ AND M. RAJAGOPALAN
}

\begin{abstract}
Let $(X, \sigma)$ be a given topological space. A compression $\tau$ of $\sigma$ is regular-invariant if and only if for every regular space $Y$ the $\sigma$-continuous functions into $Y$ are also $\tau$-continuous. $\sigma$ is regular minimal if no proper compression of $\sigma$ is regular-invariant. J. A. Guthrie and $\mathrm{H}$. E. Stone posed the problem of whether every semiregular space is regular minimal. We answer this question in the negative.
\end{abstract}

We begin by recalling a few definitions. Let $(X, \sigma)$ be a toplogical space. By a compression $\tau$ of $\sigma$ we mean a topology (on $X$ ) that is coarser than $\sigma$. A compression $\tau$ of $\sigma$ is regular-invariant if for every regular space $Y$ the $\sigma$-continuous functions into $Y$ are also $\tau$-continuous. $\sigma$ is regular minimal if no proper compression of $\sigma$ is regular-invariant. Finally a toplogical space is semiregular if it has a base of regular open sets. Katetov [2] showed that every regular minimal space is semiregular. In [1] J. A. Guthrie and H. E. Stone asked if every semiregular space is regular minimal. We shall give an example to show that this is not true in general. Let $X=[0, \infty) \times$ $[0, \infty)-\{0\} \times(0,1)$, and let $z$ denote the point $(0,0)$. We now define a topology on $X$ by specifying a base for the neighborhoods of each point of $X$ : Let the neighborhood base of the point $z$ consist of sets of the form $\{z\} \cup(0,1 / n) \times[0,1)$, $n \in N$. For every other point $p \neq z$ let the neighborhood base consist of the sets $S(p, 1 / n) \cap X, n \in N$, where $S(p, 1 / n)$ is the open disc (in $R^{2}$ with the Euclidean topology) of radius $1 / n$ and centre $p$. We denote the resulting topology on $X$ by $\sigma$. Clearly, $(X, \sigma)$ is a semiregular space.

We claim that $(X, \sigma)$ is not regular minimal: Firstly we define another topology on $X$ by specifying the sets $\{z\} \cup(0,1 / n) \times[0,1+1 / n), n \in N$, to be a base for the neighborhoods of $z$ while the neighborhood base for points $p \neq z$ is the same as for $\sigma$. Denote the resulting topology on $X$ by $\tau$. Note that $\tau$ is a compression of $\sigma$.

Now let $(Y, \mu)$ be a regular space and let $f:(X, \sigma) \rightarrow(Y, \mu)$ be a continuous function. We claim that $f$ is $\tau$-continuous.

Proof. It suffices to prove continuity of $f$ at the point $z$ only. Let $V$ be an open subset of $Y$ such that $z \in f^{-1}(V)$. Since $(Y, \mu)$ is regular there exists an open subset $W$ of $Y$ such that $f(z) \in W \subset \bar{W} \subset V$. Therefore $z \in f^{-1}(W) \subset f^{-1}(\bar{W}) \subset f^{-1}(V)$. Since $f$ is $\sigma$-continuous there exists a basic open subset $B=\{z\} \cup(0,1 / n) \times[0,1)$,

Received by the editors April 13, 1982 and, in revised form, November 9, 1982.

1980 Mathematics Subject Classification. Primary 54A10, 54D10; Secondary 54G20.

${ }^{1}$ This author is grateful to (1) the Anglo-American Corporation, South Africa and the Council for Scientific and Industrial Research, South Africa, for their grants which made this research possible and (2) the Department of Mathematics of the University of Toledo, Toledo, Ohio, where this research was done during the academic year 1981-1982. 
for some $n \in N$, such that $B \subset f^{-1}(W)$. It follows that

$$
\operatorname{cl}_{\sigma}(B)=\{z\} \cup(0,1 / n) \times[0,1] \subset \operatorname{cl}_{\sigma}\left(f^{-1}(W)\right) \subset f^{-1}(\bar{W}) \subset f^{-1}(V) .
$$

In particular, we have that the set $C=[0,1] \times\{1\} \subset f^{-1}(V)$. Therefore, for every point $p \in C$ there exists an integer $n(p) \in N$ such that $C \subset \cup_{p \in C} S(p, 1 / n(p)) \subset$ $f^{-1}(V)$. Since $C$ is compact in $(X, \sigma)$, there are a finite number of points $p_{1}$, $p_{2}, \ldots, p_{m}$ in $C$ such that

$$
C \subset \bigcup_{i=1}^{m} S\left(p_{i}, 1 / n\left(p_{i}\right)\right) .
$$

Let $r=\max \left\{n, n\left(p_{1}\right), \ldots, n\left(p_{m}\right)\right\}$. It follows that

$$
\{z\} \cup(0,1 / r) \times[0,1+1 / r) \subset f^{-1}(V),
$$

and this proves that $f$ is $\tau$-continuous at $(0,0)$.

ACKNOWLeDgement. The main result of this paper has been announced much earlier (in 1977) by Jack Porter and P. L. N. Sarma (see [4]), though their results have not appeared in print so far. The authors thank the referee for bringing [4] to their attention.

\section{REFERENCES}

1. J. A. Guthrie and H. E. Stone, Pseudocompactness and invariance of continuity, (jeneral Topology and Appl. 7 (1977), 1-13.

2. M. Katetov, Über H-abgeschlosseme und bikompakte Raume, Časopis Math. Phys. 69 (1939-1940), 36-49.

3. S. Willard, General topology, Addison-Wesley, Reading, Mass., 1970.

4. J. Porter and P. L. N. Sarma, Invariance of continuity, Notices Amer. Math. Soc. 24 (1977), A-556: Abstract \#77T-G103.

Department of Mathematics, University of Durban-Westville, Durban 4000, South Africa Department of Mathematics, University of Toledo, Toledo, OHio 43606 